\title{
Association between dietary and beverage consumption patterns in the SUN (Seguimiento Universidad de Navarra) cohort study
}

\author{
A Sánchez-Villegas ${ }^{1,2, *}$, E Toledo ${ }^{2,3}$, M Bes-Rastrollo ${ }^{2,4}, J M$ Martín-Moreno ${ }^{5}$, A Tortosa ${ }^{2}$ \\ and MA Martínez-González ${ }^{2}$ \\ ${ }^{1}$ Center for Health Sciences, Department of Clinical Sciences, University of Las Palmas de Gran Canaria, \\ PO Box 550, CP 35080, Las Palmas de Gran Canaria, Spain: ${ }^{2}$ Department of Preventive Medicine and Public \\ Health, Clínica Universitaria - Medical School, University of Navarra, Pamplona, Spain: ${ }^{3}$ Department of \\ Preventive Medicine and Quality Management, Hospital Virgen del Camino, Pamplona, Spain: ${ }^{4}$ Department of \\ Nutrition, Harvard School of Public Health, Boston, MA, USA: ${ }^{5}$ Department of Preventive Medicine and Public \\ Health, University of Valencia, Valencia, Spain
}

Submitted 20 July 2007: Accepted 25 February 2008: First published online 15 April 2008

\begin{abstract}
Objective: The objective of the present study was to determine the dietary patterns of a Mediterranean cohort and relate them to the observed patterns of beverage consumption.

Design: Prospective cohort study. Dietary habits were assessed with a semiquantitative FFQ validated in Spain. A principal components factor analysis was used to identify dietary patterns and to classify subjects according to their adherence to these patterns. The association between adherence to each dietary pattern and beverage consumption was assessed cross-sectionally. In a longitudinal analysis (2-year follow-up), the relationship between adherence to the baseline dietary patterns and the likelihood of changing alcohol consumption was ascertained.

Setting: The SUN (Seguimiento Universidad de Navarra) study is conducted in Spain. Subjects: In total, 15073 university graduates were included in the analyses.

Results: Two major dietary patterns were identified. We labelled them as 'Western dietary pattern' (WDP) and 'Mediterranean dietary pattern' (MDP). Higher adherence to the WDP was associated with higher consumption of carbonated beverages and whole-fat milk ( $P$ for trend $<0 \cdot 001$ ), while higher adherence to the MDP was associated with higher consumption of decaffeinated coffee, orange juice, other natural juices, diet carbonated drinks, low-fat milk and bottled water ( $P$ for trend $<0 \cdot 001$ ). Participants with higher adherence to the WDP were less likely to decrease their alcohol consumption during follow-up (OR between extreme quintiles $=0 \cdot 68 ; 95 \%$ CI 0.56, 0.84). By contrast, participants with higher adherence to the MDP were less likely to increase their alcohol consumption $(\mathrm{OR}=0 \cdot 66,95 \%$ CI $0 \cdot 46,0 \cdot 95)$.

Conclusion: In this cohort of university graduates, a healthier dietary pattern was associated with a healthier pattern of beverage consumption.
\end{abstract}

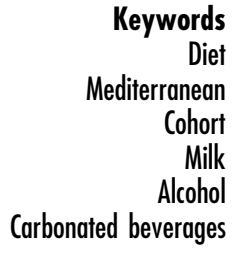

In the examination of the relationship between diet and disease, interest has drifted from the study of single nutrients to analysis of the adherence to different dietary patterns. In this context, two different food patterns have been defined because they are related to the risk of different diseases: (i) a protective 'prudent pattern' rich in fruits, vegetables, fish, poultry and whole grains; and (ii) a deleterious 'Western' pattern rich in red meat, processed meat, French fries, high-fat dairy products, refined grains, and sweets and desserts ${ }^{(1)}$. In southern Europe, interest has focused on the so-called 'Mediterranean diet', similar to the 'prudent pattern' but rich in olive oil ${ }^{(2)}$, which has also been associated with a decreased risk of CVD, cancer and other illnesses ${ }^{(3,4)}$. In contrast, although several studies have reported major dietary patterns among adults, very little information discussing dietary patterns among young people or analysing beverage patterns is available.

As far as beverage consumption is concerned, in the context of the current overweight and obesity epidemic, dietary strategies recommend a water intake of 1-2 litres 
daily and a higher consumption of beverages with no or little energy than of beverages with more energy ${ }^{(5)}$. However, according to current beverage patterns, water is being replaced by other less healthy, high-energy beverages. In the US population, the consumption of soft drinks has risen ${ }^{(6)}$. This increase has also been observed in Europe and, specifically, in Spain where the consumption of sugar-sweetened soft drinks has grown by $21 \%$ from 1991 to $2001^{(7)}$.

Sugar-sweetened soft drinks have been shown to be associated with a higher risk of weight gain ${ }^{(8-10)}$. Other beverages, such as red wine and tea, have been reported to have some potentially beneficial effects on vascular reactivity ${ }^{(11)}$. Furthermore, moderate consumption of red wine has been associated with a reduced risk of type 2 diabetes mellitus ${ }^{(12)}$.

Some authors have linked beverage and dietary patterns in order to determine a relationship between them, finding that subjects with healthier food patterns have also healthier beverage patterns ${ }^{(13)}$.

The aim of the present study was to assess the adherence to different dietary patterns of a free-living Mediterranean cohort of university graduates and to relate them to their beverage consumption patterns.

\section{Materials and methods}

\section{Study population}

The SUN (Seguimiento Universidad de Navarra) study is a prospective cohort study based on university graduates and designed in collaboration with the Harvard School of Public Health. Its methodology is similar to that used in the two large American cohorts, the Nurses' Health Study and the Health Professionals' Follow-up study. Information is collected using self-administered questionnaires sent by postal mail every two years. The recruitment of participants started in December 1999 and is permanently ongoing, as this is a dynamic cohort study. Up to January 2007, 16431 participants had answered both the baseline and the first 2-year follow-up questionnaire (hereafter, baseline = Q_0 and follow-up = Q_2).

Those participants who reported extremely low or high values for total energy intake $(<2.51 \mathrm{MJ} / \mathrm{d}(<600 \mathrm{kcal} / \mathrm{d})$ in men and $<1.67 \mathrm{MJ} / \mathrm{d}(<400 \mathrm{kcal} / \mathrm{d})$ in women or $>17.57 \mathrm{MJ} / \mathrm{d}(>4200 \mathrm{kcal} / \mathrm{d})$ in men and $>14.64 \mathrm{MJ} / \mathrm{d}$ $(>3500 \mathrm{kcal} / \mathrm{d})$ in women; $n$ 1358) were excluded. Finally, data from 15073 participants remained available for the analysis.

The Institutional Review Board of the University of Navarra (Clínica Universitaria) approved the study protocol. Voluntary completion of the first self-administered questionnaire was considered to imply informed consent.

\section{Dietary patterns assessment}

Dietary habits were ascertained at baseline (Q_0) through a semi-quantitative FFQ (136 food items) previously

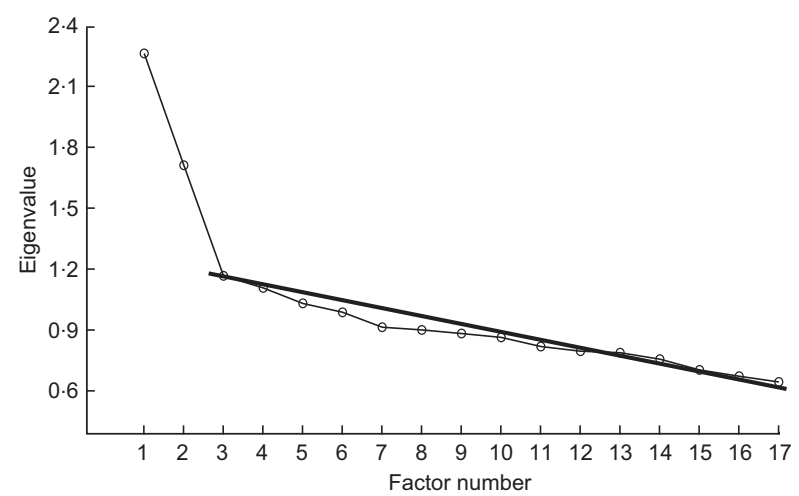

Fig. 1 Scree plot of eigenvalues plotted against their factors to identify the number of factors to be extracted

validated in Spain ${ }^{(14)}$. Nutrient scores were calculated as frequency multiplied by nutrient composition of specified portion sizes, where frequencies were measured in nine categories $(6+$ times daily/4-6 times daily/2-3 times daily/once daily/5-6 times weekly/2-4 times weekly/ once weekly/1-3 times monthly/never or almost never) for each food item. Nutrient intake scores were computed using an ad hoc computer program developed specifically for this aim. A trained dietitian updated the nutrient databank using the latest available information in food composition tables for Spain ${ }^{(15,16)}$.

The 136 food items included in the semi-quantitative FFQ were grouped into twenty-five predefined food categories. A principal components analysis based on the stratified food groups was conducted to identify the major dietary patterns in the cohort ${ }^{(17)}$. The food groups 'other fats' and 'cooked potatoes' were excluded for the subsequent analyses because their measures of sampling adequacy were lower than $0 \cdot 70$. The approach used to determine the number of factors to be extracted was the scree plot examination ${ }^{(17)}$. This method consists of plotting the extracted factors against their eigenvalues to identify distinct breaks in the slope of the plot. To determine where the break occurs, a straight line is drawn through the lower eigenvalues. That point where the factors curve above the line identifies the number of factors to be extracted (Fig. 1). Afterwards, the two obtained factors were rotated using the Varimax orthogonal rotation.

We used the factor loading matrix to extract the weights (factor loadings) for each food group. Food groups such as 'butter', 'margarine', 'home-made pastries', 'chocolate and sweets', 'sugar' and 'legumes', with factor loadings lower than $0 \cdot 30$, were excluded from the final model ${ }^{(18)}$. After considering the weights of the food groups in each factor, we labelled the first factor as 'Western dietary pattern' (WDP) and the second factor as 'Mediterranean dietary pattern' (MDP) (Table 1). These variables were calculated as linear combinations of the standardized intake of the seventeen remaining food groups weighted 
Table 1 Factor loading matrix for the two major dietary patterns identified by using food consumption data: the SUN (Seguimiento Universidad de Navarra) prospective cohort study

\begin{tabular}{|c|c|c|}
\hline & \multicolumn{2}{|c|}{ Dietary pattern } \\
\hline & Western & Mediterranean \\
\hline Processed meat products & 0.55 & \\
\hline Red meat & 0.57 & \\
\hline Poultry & & 0.32 \\
\hline Fast food & $0 \cdot 60$ & \\
\hline Eggs & 0.42 & \\
\hline Fish and seafood & & 0.55 \\
\hline Low-fat dairy products* & & 0.45 \\
\hline Whole-fat dairy products* & $0 \cdot 37$ & \\
\hline Whole-wheat bread & & 0.35 \\
\hline Nuts & & $0 \cdot 30$ \\
\hline Processed meals & 0.40 & \\
\hline Processed pastries & 0.39 & \\
\hline Sauces & 0.43 & \\
\hline Refined grains & 0.33 & \\
\hline Vegetables & & 0.69 \\
\hline Fruits & & 0.59 \\
\hline Olive oil & & 0.34 \\
\hline
\end{tabular}

Absolute values $<0.30$ were not included in the table.

The first factor explained $13.3 \%$ of the total variance and the second factor explained $10 \cdot 1 \%$ of the total variance.

*Milk can be considered a beverage so it has been excluded from this food grouping.

by their factor score coefficients. These coefficients were generated automatically by the statistical software ${ }^{(17)}$. Finally, the continuous variables thus built were categorized into quintiles.

\section{Beverages assessment}

The baseline FFQ (Q_0) also collected information about habits of beverage consumption and included specific items for spirits, total wine, red wine, beer, soda drinks, diet drinks, coffee, decaffeinated coffee, natural orange juice, other natural juices, canned juices, whole-fat and low-fat milk, tap water and bottled water.

\section{Assessment of changes in dietary babits}

The follow-up questionnaire (Q_2) included questions regarding change in the consumption of several food items (dairy products, meat, fish, butter, olive oil, vegetables and alcohol) since Q_0 (no change, increase or decrease in consumption).

\section{Assessment of other variables}

The baseline assessment (Q_0) included other questions as well. Sociodemographic (e.g. gender, age, marital status), anthropometric (e.g. weight and height), lifestyle and health-related habits (e.g. smoking status and physical activity during leisure time) and medical history variables (e.g. prevalence of chronic diseases such as cancer, CVD or ulcer) were collected.

The physical activity questionnaire included information about seventeen activities including walking, jogging, bicycling, static bicycling, swimming, racquet sports, soccer, aerobic, judo, trekking, skiing, sailing and gardening.
To quantify the volume of activity during leisure time, an activity metabolic energy equivalent task (MET) index was computed by assigning a multiple of the resting metabolic rate (MET score) to each activity ${ }^{(19)}$, the time spent in each of the activities was multiplied by the MET score specific to each activity, and then the MET scores were summed over all activities to obtain a value of overall weekly MET-hours. In the validation study carried out in a sub-sample of the cohort, there was a significant correlation between the physical activity measured objectively through an accelerometer and the overall weekly MET-hours assessed using this questionnaire $(r=0.51, P<0 \cdot 001)^{(20)}$.

Participants were classified as suffering from CVD at baseline if they had reported at least one of the following conditions: myocardial infarction, stroke, atrial fibrillation, paroxysmal tachycardia, coronary artery bypass grafting or other revascularization procedures, heart failure, aortic aneurism, pulmonary embolism or peripheral venous thrombosis.

\section{Statistical analysis}

Linear regression models were used to assess the association between adherence to the identified dietary intake profiles and the pattern of beverage consumption in Q_0. Tests of linear trend across increasing quintiles of dietary pattern adherence were calculated for each type of beverage. For that purpose, the median value of adherence was imputed for each quintile of adherence.

Non-conditional logistic regression models were fit to assess the relationship between adherence to the identified dietary patterns and the likelihood of changing alcohol consumption over two years in our cohort (reported in Q_2). The reference category was no change in consumption. Odds ratios and their 95\% confidence intervals were calculated by considering the lowest quintile of adherence as the reference category. Potential confounders included in both multivariate models (linear and logistic) were: gender, age, BMI, physical activity during leisure time, smoking, presence of any severe disease at baseline (cancer, CVD or ulcer) and total energy intake.

\section{Results}

Table 2 shows the main characteristics of the participants according to extreme quintiles of WDP and MDP adherence. WDP adherence was higher among younger participants, men, smokers and single persons.

Participants belonging to the highest quintile of MDP were older, physically more active, and more likely to be women, married subjects and ex-smokers. Moreover, the history of diseases such as CVD, cancer or ulcer was higher among those subjects with higher adherence to this dietary pattern.

Tables 3 and 4 show the associations between quintiles of WDP and MDP adherence and the baseline consumption 
Table 2 Main characteristics of participants according to extreme quintiles of adherence ${ }^{\star}$ to the defined dietary patterns: the SUN (Seguimiento Universidad de Navarra) prospective cohort study

\begin{tabular}{|c|c|c|c|c|c|c|c|c|c|c|}
\hline & \multicolumn{5}{|c|}{ Western dietary pattern } & \multicolumn{5}{|c|}{ Mediterranean dietary pattern } \\
\hline & \multicolumn{2}{|c|}{ Q1 } & \multicolumn{2}{|c|}{ Q5 } & \multirow[b]{2}{*}{$P+$} & \multicolumn{2}{|c|}{ Q1 } & \multicolumn{2}{|c|}{ Q5 } & \multirow[b]{2}{*}{$P+$} \\
\hline & Mean & SD & Mean & SD & & Mean & SD & Mean & SD & \\
\hline$n$ & \multicolumn{2}{|c|}{3014} & \multicolumn{2}{|c|}{3014} & & \multicolumn{2}{|c|}{3014} & \multicolumn{2}{|c|}{3014} & \\
\hline Age (years) & $42 \cdot 2$ & $13 \cdot 2$ & $34 \cdot 5$ & $11 \cdot 2$ & $<0.001$ & $36 \cdot 1$ & $11 \cdot 8$ & 39.9 & $13 \cdot 0$ & $<0.001$ \\
\hline Baseline BMI $\left(\mathrm{kg} / \mathrm{m}^{2}\right)$ & $23 \cdot 7$ & $3 \cdot 7$ & $23 \cdot 7$ & $3 \cdot 6$ & 0.78 & $23 \cdot 5$ & $3 \cdot 7$ & $23 \cdot 6$ & $3 \cdot 6$ & $0 \cdot 13$ \\
\hline $\begin{array}{l}\text { Physical activity during leisure } \\
\text { time (MET-h/week) }\end{array}$ & $24 \cdot 0$ & $22 \cdot 5$ & $25 \cdot 0$ & $23 \cdot 2$ & $0 \cdot 10$ & $20 \cdot 4$ & $18 \cdot 9$ & $27 \cdot 7$ & $26 \cdot 5$ & $<0.001$ \\
\hline Energy intake $(\mathrm{MJ} / \mathrm{d})$ & $7 \cdot 44$ & $2 \cdot 32$ & $12 \cdot 79$ & $2 \cdot 03$ & $<0.001$ & $8 \cdot 50$ & $2 \cdot 79$ & $11 \cdot 41$ & $2 \cdot 44$ & $<0.001$ \\
\hline Energy intake (kcal/d) & $1777 \cdot 8$ & $555 \cdot 6$ & $3057 \cdot 5$ & $485 \cdot 0$ & $<0.001$ & $2031 \cdot 4$ & $666 \cdot 8$ & $2725 \cdot 9$ & $584 \cdot 0$ & $<0.001$ \\
\hline \multicolumn{11}{|l|}{ Gender (\%) } \\
\hline Women & \multirow{2}{*}{\multicolumn{2}{|c|}{$65 \cdot 0$}} & \multirow{2}{*}{\multicolumn{2}{|c|}{$42 \cdot 9$}} & $<0.001 \ddagger$ & \multirow{2}{*}{\multicolumn{2}{|c|}{$46 \cdot 9$}} & \multirow{2}{*}{\multicolumn{2}{|c|}{$68 \cdot 6$}} & $<0.001 \ddagger$ \\
\hline Marital status (\%) & & & & & $<0.001 \ddagger$ & & & & & $<0.001 \ddagger$ \\
\hline Single & \multicolumn{2}{|c|}{$39 \cdot 4$} & \multicolumn{2}{|c|}{$56 \cdot 5$} & & \multicolumn{2}{|c|}{$52 \cdot 2$} & \multicolumn{2}{|c|}{$41 \cdot 9$} & \\
\hline Married & \multicolumn{2}{|c|}{$53 \cdot 5$} & \multicolumn{2}{|c|}{$41 \cdot 3$} & & \multicolumn{2}{|c|}{$43 \cdot 8$} & \multicolumn{2}{|c|}{$52 \cdot 5$} & \\
\hline Divorced & \multicolumn{2}{|c|}{$3 \cdot 8$} & \multicolumn{2}{|c|}{0.9} & & \multicolumn{2}{|c|}{$2 \cdot 0$} & \multicolumn{2}{|c|}{$2 \cdot 9$} & \\
\hline Widowed & \multirow{2}{*}{\multicolumn{2}{|c|}{$\begin{array}{l}1 \cdot 9 \\
1 \cdot 3\end{array}$}} & & & & & & & & \\
\hline Other & & & 0 & & & & & & & \\
\hline Smoking (\%) & & & & & $<0.001 \ddagger$ & & & & & $<0.001 \ddagger$ \\
\hline Never smoker & 43 & & 49 & & & 48 & & 45 & & \\
\hline Ex-smoker $>10$ years & 17 & & & & & & & 15 & & \\
\hline Ex-smoker $3-9$ years & 11 & & & & & & & 10 & & \\
\hline Ex-smoker $<3$ years & 8 & 5 & & & & & & & & \\
\hline Current smoker & 19 & & 28 & & & 27 & & 20 & & \\
\hline History of diseases (\%) & & & & & & & & & & \\
\hline Cardiovascular & 6 & 1 & 3 & & $<0.001 \ddagger$ & & & & 2 & $<0.001 \ddagger$ \\
\hline Cancer & & 0 & & & $<0.001 \ddagger$ & & & & & $0.003 \ddagger$ \\
\hline Ulcer & 6 & 7 & & & $0.002 \ddagger$ & & 5 & & 5 & $0.08 \ddagger$ \\
\hline
\end{tabular}

${ }^{*} \mathrm{Q} 1,1$ st quintile (lowest); Q5, 5th quintile (highest). $+P$ from Student $t$ test.

$\ddagger P$ from $\chi^{2}$ test.

of several beverages in the cohort. Subjects in the lowest quintile of adherence were considered as the reference category. Higher adherence to the WDP was associated with higher consumption of sugared soda drinks (mean consumption $(\mathrm{g} / \mathrm{d}): 22 \cdot 7,24 \cdot 8,28 \cdot 2,30 \cdot 4$ and $43 \cdot 8$ for increasing quintiles, $P$ for trend $<0 \cdot 001$ ) and whole-fat milk (mean consumption (g/d): 47.5, 61.1, 65.9, 67.7 and $71 \cdot 1$ for increasing quintiles, $P$ for trend $<0 \cdot 001)$. Furthermore, the consumption of other beverages considered healthy beverages, such as decaffeinated coffee, orange and other natural juices, diet soda drinks and low-fat milk, was lower among those subjects with high adherence to this pattern $(P$ for trend $<0 \cdot 001$ in all analyses). The consumption of tap water increased monotonically while the consumption of bottled water decreased significantly across increasing quintiles of adherence to the WDP (Table 3). Consumption of alcoholic beverages such as spirits, wine and beer was lower in the upper quintiles of WDP adherence although a non-significant linear trend was observed for spirits consumption ( $P$ for trend $<0.001$ for wine and beer, $P$ for trend $=0 \cdot 165$ for spirits).

In our cohort, the consumption of healthy beverages such as decaffeinated coffee, orange and other natural juices, sugarless soda drinks, low-fat milk and also of bottled water was higher among subjects with higher adherence to the MDP (Table 4). An inverse statistically significant dose-response relationship was found for whole-fat milk, soda drinks and alcoholic beverages, except red wine, and MDP adherence (red wine, mean consumption $(\mathrm{g} / \mathrm{d}): 25 \cdot 3,26 \cdot 9,26 \cdot 2,28 \cdot 1$ and $22 \cdot 4$ for increasing quintiles, $P$ for trend $=0 \cdot 105$ ).

Participants with high adherence to the WDP showed a lower probability of decreasing alcohol consumption during the first two years of follow-up. Specifically, the multivariate $\mathrm{OR}$ of decreasing alcohol consumption for the highest quintile of adherence to the WDP was 0.68 (95\% CI 0.56, 0.84). On the contrary, participants with high adherence to the MDP showed a higher probability of decreasing alcohol consumption (adjusted $\mathrm{OR}=1 \cdot 31$; $95 \%$ CI $1 \cdot 11,1 \cdot 56$ ).

\section{Discussion}

Two major dietary patterns were found in the present analysis of the first 15073 participants of the SUN cohort study with 2 years of follow-up. This finding is consistent with a previous report of our group assessing 3847 university graduates $^{(21)}$. Adherence to a Western diet (WDP) was associated with the consumption of several kinds of beverages such as sugar-sweetened soft drinks and 
Table 3 Consumption of beverages according to quintiles of adherence* to the Western dietary pattern: the SUN (Seguimiento Universidad de Navarra) prospective cohort study

Western dietary pattern

\begin{tabular}{|c|c|c|c|c|c|c|c|c|c|c|c|}
\hline & \multirow{2}{*}{\multicolumn{2}{|c|}{ Q1 }} & \multirow{2}{*}{\multicolumn{2}{|c|}{ Q2 }} & \multirow{2}{*}{\multicolumn{2}{|c|}{ Q3 }} & & & & & \\
\hline & & & & & & & \multicolumn{2}{|c|}{ Q4 } & \multicolumn{2}{|c|}{ Q5 } & \multirow[b]{2}{*}{$P$ for trend } \\
\hline & Mean & $95 \% \mathrm{Cl}$ & Mean & $95 \% \mathrm{Cl}$ & Mean & $95 \% \mathrm{Cl}$ & Mean & $95 \% \mathrm{Cl}$ & Mean & $95 \% \mathrm{Cl}$ & \\
\hline Total alcohol consumption $(\mathrm{g} / \mathrm{d})$ & $5 \cdot 7$ & $5 \cdot 4,6 \cdot 1$ & $5 \cdot 8$ & $5 \cdot 3,6 \cdot 3$ & $5 \cdot 3$ & $4 \cdot 8,5 \cdot 9$ & $4 \cdot 7$ & $4 \cdot 1,5 \cdot 2$ & $3 \cdot 6$ & $2 \cdot 9,4 \cdot 3$ & $<0.001$ \\
\hline Spirits $(g / d)$ & $2 \cdot 8$ & $2 \cdot 5,3 \cdot 1$ & $3 \cdot 2$ & $2 \cdot 7,3 \cdot 7$ & $2 \cdot 8$ & $2 \cdot 2,3 \cdot 3$ & $2 \cdot 5$ & $1.9,3.0$ & $2 \cdot 6$ & $1 \cdot 9,3 \cdot 2$ & 0.165 \\
\hline Total wine $(\mathrm{g} / \mathrm{d})$ & $32 \cdot 2$ & $29 \cdot 7,34 \cdot 8$ & $32 \cdot 4$ & $28 \cdot 7,36 \cdot 1$ & $29 \cdot 9$ & $26 \cdot 0,33 \cdot 8$ & $25 \cdot 0$ & $20 \cdot 9,29 \cdot 2$ & 18.8 & $13 \cdot 9,23 \cdot 7$ & $<0.001$ \\
\hline Red wine $(\mathrm{g} / \mathrm{d})$ & $27 \cdot 2$ & $24 \cdot 9,29 \cdot 6$ & $27 \cdot 7$ & $24 \cdot 5,30 \cdot 9$ & $25 \cdot 6$ & $22 \cdot 2,29 \cdot 0$ & $22 \cdot 0$ & $18 \cdot 3,25 \cdot 7$ & $16 \cdot 3$ & $12 \cdot 0,20 \cdot 5$ & $<0.001$ \\
\hline $\operatorname{Beer}(\mathrm{g} / \mathrm{d})$ & $45 \cdot 2$ & $41 \cdot 5,48 \cdot 8$ & $43 \cdot 7$ & $37 \cdot 7,49 \cdot 6$ & $41 \cdot 4$ & $35 \cdot 2,47 \cdot 6$ & 38.0 & $31 \cdot 3,44 \cdot 7$ & $25 \cdot 6$ & $17 \cdot 8,33 \cdot 4$ & $<0.001$ \\
\hline Carbonated drinks (g/d) & $22 \cdot 7$ & $20 \cdot 4,25 \cdot 0$ & $24 \cdot 8$ & $20 \cdot 6,29 \cdot 1$ & $28 \cdot 2$ & $23 \cdot 7,32 \cdot 6$ & $30 \cdot 4$ & $25 \cdot 5,35 \cdot 2$ & 43.8 & $38 \cdot 2,49 \cdot 4$ & $<0.001$ \\
\hline Diet drinks $(\mathrm{g} / \mathrm{d})$ & $25 \cdot 0$ & $21 \cdot 8,28 \cdot 2$ & $20 \cdot 2$ & $16 \cdot 0,24 \cdot 3$ & $16 \cdot 0$ & $11 \cdot 7,20 \cdot 4$ & $17 \cdot 0$ & $12 \cdot 3,21 \cdot 7$ & $12 \cdot 7$ & $7 \cdot 2,18 \cdot 2$ & $<0.001$ \\
\hline Coffee $(\mathrm{g} / \mathrm{d})$ & $58 \cdot 6$ & $56 \cdot 3,60 \cdot 9$ & $59 \cdot 1$ & $55 \cdot 8,62 \cdot 4$ & $56 \cdot 8$ & $53 \cdot 4,60 \cdot 3$ & $56 \cdot 0$ & $52 \cdot 3,59 \cdot 8$ & $51 \cdot 1$ & $46 \cdot 7,55 \cdot 4$ & $<0.001$ \\
\hline Decaffeinated coffee $(\mathrm{g} / \mathrm{d})$ & $15 \cdot 5$ & $14 \cdot 3,16 \cdot 7$ & $12 \cdot 1$ & $10 \cdot 5,13 \cdot 7$ & $11 \cdot 4$ & $9 \cdot 7,13 \cdot 1$ & $10 \cdot 6$ & $8 \cdot 8,12 \cdot 4$ & 6.5 & $4 \cdot 4,8 \cdot 5$ & $<0.001$ \\
\hline Natural orange juice $(\mathrm{g} / \mathrm{d})$ & $60 \cdot 8$ & $56 \cdot 8,64 \cdot 9$ & $46 \cdot 1$ & $41 \cdot 3,50 \cdot 9$ & $33 \cdot 4$ & $28 \cdot 4,38 \cdot 5$ & $22 \cdot 6$ & $17 \cdot 1,28 \cdot 1$ & 3.9 & $0,55 \cdot 2$ & $<0.001$ \\
\hline Other natural juices ( $\mathrm{g} / \mathrm{d})$ & $12 \cdot 4$ & $10 \cdot 5,14 \cdot 3$ & $8 \cdot 7$ & $6 \cdot 4,11 \cdot 0$ & $4 \cdot 4$ & $2 \cdot 0,6 \cdot 8$ & 0.8 & $0,3 \cdot 4$ & 0 & 0,0 & $<0.001$ \\
\hline Canned juices (g/d) & $17 \cdot 3$ & $15 \cdot 1,19 \cdot 5$ & $14 \cdot 8$ & $11 \cdot 5,18 \cdot 1$ & $14 \cdot 1$ & $10 \cdot 6,17 \cdot 5$ & $13 \cdot 9$ & $10 \cdot 1,17 \cdot 6$ & $10 \cdot 1$ & $5 \cdot 7,14 \cdot 4$ & 0.002 \\
\hline Whole-fat milk (g/d) & $47 \cdot 5$ & $43 \cdot 3,51 \cdot 8$ & $61 \cdot 1$ & $52 \cdot 8,69 \cdot 4$ & 65.9 & $57 \cdot 1,59 \cdot 6$ & $67 \cdot 7$ & $62 \cdot 3,81 \cdot 2$ & $71 \cdot 1$ & $60 \cdot 2,82 \cdot 0$ & $<0.001$ \\
\hline Low-fat milk $(\mathrm{g} / \mathrm{d})$ & $200 \cdot 6$ & $192 \cdot 5,208 \cdot 7$ & $174 \cdot 5$ & $163 \cdot 3,185 \cdot 7$ & $140 \cdot 6$ & $128 \cdot 9,152 \cdot 3$ & $110 \cdot 4$ & $97 \cdot 7,123 \cdot 0$ & $44 \cdot 1$ & $29 \cdot 5,58 \cdot 9$ & $<0.001$ \\
\hline Tap water $(\mathrm{g} / \mathrm{d})$ & $502 \cdot 3$ & $484 \cdot 2,520 \cdot 5$ & $536 \cdot 4$ & $492 \cdot 5,580 \cdot 5$ & $538 \cdot 4$ & $493 \cdot 2,583 \cdot 8$ & $566 \cdot 8$ & $519 \cdot 4,614 \cdot 3$ & 559.8 & $507 \cdot 6,612 \cdot 1$ & $<0.001$ \\
\hline Bottled water $(\mathrm{g} / \mathrm{d})$ & $311 \cdot 0$ & $294 \cdot 2,327 \cdot 8$ & $251 \cdot 5$ & $212 \cdot 1,290 \cdot 2$ & $214 \cdot 6$ & $174 \cdot 4,254 \cdot 8$ & $179 \cdot 7$ & $137 \cdot 6,221 \cdot 8$ & $147 \cdot 1$ & $101 \cdot 0,192 \cdot 3$ & $<0.001$ \\
\hline
\end{tabular}

Adjusted for age (years), gender, energy intake (MJ/d), BMI (four categories), physical activity during leisure time (quartiles, MET-h/week), smoking (six categories), marital status (four categories) and several diseases (CVD, cancer and ulcer).

${ }^{*}$ Q1-Q5, 1st quintile (lowest)-5th quintile (highest).

Table 4 Consumption of beverages according to quintiles of adherence* to the Mediterranean dietary pattern: the SUN (Seguimiento Universidad de Navarra) prospective cohort study

\begin{tabular}{|c|c|c|c|c|c|c|c|c|c|c|c|}
\hline & \multicolumn{11}{|c|}{ Mediterranean dietary pattern } \\
\hline & \multicolumn{2}{|c|}{ Q1 } & \multicolumn{2}{|c|}{ Q2 } & \multicolumn{2}{|c|}{ Q3 } & \multicolumn{2}{|c|}{ Q4 } & \multicolumn{2}{|c|}{ Q5 } & \multirow[b]{2}{*}{$P$ for trend } \\
\hline & Mean & $95 \% \mathrm{Cl}$ & Mean & $95 \% \mathrm{Cl}$ & Mean & $95 \% \mathrm{Cl}$ & Mean & $95 \% \mathrm{Cl}$ & Mean & $95 \% \mathrm{Cl}$ & \\
\hline Total alcohol consumption $(\mathrm{g} / \mathrm{d})$ & $6 \cdot 6$ & $6 \cdot 2,7 \cdot 0$ & $6 \cdot 9$ & $6 \cdot 4,7 \cdot 4$ & $6 \cdot 6$ & $6 \cdot 1,7 \cdot 1$ & $6 \cdot 5$ & $6 \cdot 0,7 \cdot 0$ & $5 \cdot 1$ & $4 \cdot 6,5 \cdot 7$ & $<0.001$ \\
\hline Spirits $(g / d)$ & 4.5 & $4 \cdot 0,4 \cdot 9$ & 4.5 & $4 \cdot 0,5 \cdot 0$ & $4 \cdot 6$ & $4 \cdot 1,5 \cdot 2$ & $4 \cdot 1$ & $3 \cdot 5,4 \cdot 6$ & $3 \cdot 7$ & $3 \cdot 2,4 \cdot 3$ & 0.001 \\
\hline Total wine $(\mathrm{g} / \mathrm{d})$ & $30 \cdot 1$ & $27 \cdot 4,32 \cdot 7$ & $31 \cdot 6$ & $28 \cdot 0,35 \cdot 3$ & $30 \cdot 8$ & $27 \cdot 0,34 \cdot 5$ & $33 \cdot 0$ & $29 \cdot 2,36 \cdot 8$ & $25 \cdot 0$ & $20 \cdot 9,29 \cdot 0$ & 0.012 \\
\hline Red wine $(\mathrm{g} / \mathrm{d})$ & $25 \cdot 3$ & $22 \cdot 9,27 \cdot 6$ & $26 \cdot 9$ & $23 \cdot 7,30 \cdot 1$ & $26 \cdot 2$ & $23 \cdot 0,21 \cdot 0$ & $28 \cdot 1$ & $24 \cdot 7,31 \cdot 4$ & $22 \cdot 4$ & $18 \cdot 9,26 \cdot 0$ & $0 \cdot 105$ \\
\hline $\operatorname{Beer}(\mathrm{g} / \mathrm{d})$ & $57 \cdot 2$ & $52 \cdot 8,61 \cdot 7$ & $61 \cdot 0$ & $55 \cdot 1,66 \cdot 8$ & $54 \cdot 4$ & $48 \cdot 5,60 \cdot 4$ & $52 \cdot 4$ & $46 \cdot 3,58 \cdot 6$ & 38.9 & $32 \cdot 5,45 \cdot 4$ & $<0.001$ \\
\hline Carbonated drinks ( $\mathrm{g} / \mathrm{d})$ & $60 \cdot 3$ & $56 \cdot 1,64 \cdot 5$ & $43 \cdot 3$ & $39 \cdot 2,47 \cdot 5$ & $38 \cdot 4$ & $34 \cdot 1,42 \cdot 6$ & $28 \cdot 2$ & $23 \cdot 9,32 \cdot 6$ & $17 \cdot 6$ & $13 \cdot 0,22 \cdot 1$ & $<0.001$ \\
\hline Diet drinks $(\mathrm{g} / \mathrm{d})$ & $16 \cdot 7$ & $13 \cdot 8,19 \cdot 6$ & $16 \cdot 3$ & $12 \cdot 2,13 \cdot 1$ & $22 \cdot 3$ & $18 \cdot 1,26 \cdot 5$ & $25 \cdot 8$ & $21 \cdot 5,30 \cdot 1$ & 37.8 & $33 \cdot 3,42 \cdot 3$ & $<0.001$ \\
\hline Coffee $(\mathrm{g} / \mathrm{d})$ & $57 \cdot 5$ & $55 \cdot 3,59 \cdot 8$ & $59 \cdot 6$ & $56 \cdot 3,62 \cdot 8$ & $60 \cdot 1$ & $56 \cdot 8,63 \cdot 4$ & $58 \cdot 3$ & $54 \cdot 9,61 \cdot 7$ & $56 \cdot 7$ & $53 \cdot 1,60 \cdot 3$ & 0.336 \\
\hline Decaffeinated coffee $(\mathrm{g} / \mathrm{d})$ & $9 \cdot 9$ & $8 \cdot 9,10 \cdot 9$ & $10 \cdot 3$ & $8 \cdot 8,11.9$ & $10 \cdot 3$ & $8 \cdot 7,11 \cdot 9$ & $12 \cdot 2$ & $10 \cdot 6,13 \cdot 8$ & $14 \cdot 1$ & $12 \cdot 4,15 \cdot 8$ & $<0.001$ \\
\hline Natural orange juice $(\mathrm{g} / \mathrm{d})$ & $36 \cdot 2$ & $33 \cdot 0,39 \cdot 3$ & $43 \cdot 7$ & $38.9,48 \cdot 5$ & $49 \cdot 2$ & $44 \cdot 3,54 \cdot 1$ & $57 \cdot 0$ & $52 \cdot 0,62 \cdot 0$ & $65 \cdot 8$ & $60 \cdot 5,71 \cdot 0$ & $<0.001$ \\
\hline Other natural juices (g/d) & $8 \cdot 0$ & $6 \cdot 6,9 \cdot 3$ & $7 \cdot 4$ & $5 \cdot 2,9 \cdot 7$ & $9 \cdot 0$ & $6 \cdot 7,11 \cdot 3$ & 11.9 & $9 \cdot 6,14 \cdot 3$ & $16 \cdot 1$ & $13 \cdot 6,18 \cdot 6$ & $<0.001$ \\
\hline Canned juices $(\mathrm{g} / \mathrm{d})$ & $22 \cdot 0$ & $19 \cdot 8,24 \cdot 3$ & $20 \cdot 4$ & $17 \cdot 2,23 \cdot 7$ & $20 \cdot 6$ & $17 \cdot 2,23 \cdot 9$ & $18 \cdot 3$ & $14 \cdot 9,21 \cdot 8$ & $17 \cdot 7$ & $14 \cdot 1,21 \cdot 3$ & 0.011 \\
\hline Whole-fat milk (g/d) & $131 \cdot 5$ & $124 \cdot 6,138 \cdot 3$ & $96 \cdot 3$ & $88 \cdot 3,104 \cdot 3$ & $74 \cdot 3$ & $66 \cdot 1,82 \cdot 5$ & $50 \cdot 6$ & $42 \cdot 2,59 \cdot 0$ & $15 \cdot 0$ & $6 \cdot 2,23 \cdot 9$ & $<0.001$ \\
\hline Low-fat milk (g/d) & $123 \cdot 3$ & $116 \cdot 2,130 \cdot 3$ & $149 \cdot 2$ & $138 \cdot 1,160 \cdot 3$ & $164 \cdot 5$ & $153 \cdot 1,175 \cdot 8$ & $180 \cdot 9$ & $169 \cdot 2,192 \cdot 5$ & $210 \cdot 4$ & $198 \cdot 2,222 \cdot 7$ & $<0.001$ \\
\hline Tap water $(\mathrm{g} / \mathrm{d})$ & $549 \cdot 2$ & $531 \cdot 6,566 \cdot 7$ & $606 \cdot 7$ & $563 \cdot 7,649 \cdot 6$ & $591 \cdot 2$ & $547 \cdot 6,634 \cdot 7$ & $582 \cdot 5$ & $538 \cdot 1,626 \cdot 8$ & $585 \cdot 6$ & $539 \cdot 7,631 \cdot 4$ & $0 \cdot 213$ \\
\hline Bottled water $(\mathrm{g} / \mathrm{d})$ & $201 \cdot 1$ & $187 \cdot 4,214 \cdot 7$ & $216 \cdot 6$ & $181 \cdot 1,252 \cdot 0$ & $257 \cdot 6$ & $221 \cdot 6,293 \cdot 6$ & $306 \cdot 2$ & $269 \cdot 5,342 \cdot 8$ & $371 \cdot 2$ & $333 \cdot 2,409 \cdot 1$ & $<0.001$ \\
\hline
\end{tabular}

Adjusted for age (years), gender, energy intake (MJ/d), BMI (four categories), physical activity during leisure time (quartiles, MET-h/week), smoking (six categories), marital status (four categories) and several diseases (CVD, cancer and ulcer).

${ }^{*}$ Q1-Q5, 1st quintile (lowest)-5th quintile (highest). 
whole-fat milk. On the other hand, adherence to a Mediterranean diet (MDP) was associated with the consumption of healthier beverages such as decaffeinated coffee, orange and other natural juices, diet soda drinks and low-fat milk.

The WDP identified in our cohort shares common characteristics with those found in other large cohort studies such as the Nurses' Health Study and the Health Professionals' Follow-up Study ${ }^{(1)}$. These patterns are characterized by a high consumption of certain food items such as red and processed meats, refined grains, sweets and desserts. The harmful effect of the so-called Western diet has been documented in several studies. Recently, Esmaillzadeh et al. reported that women in the highest quintile of adherence to the WDP had greater probability of developing the metabolic syndrome (OR $=1 \cdot 68 ; 95 \%$ CI $1 \cdot 10,1.95)$ compared with women in the lowest quintile $^{(22)}$. Deleterious effects of this pattern have also been reported for other disorders such as cancer ${ }^{(23,24)}$, $\mathrm{CVD}^{(25-27)}$, obesity ${ }^{(28)}$ and diabetes ${ }^{(29)}$, as well as for different biomarkers of pathological conditions ${ }^{(30-32)}$.

The defined MDP found in the present study is characterized by a high consumption of low-fat dairy products, fish, whole-wheat bread, nuts, vegetables, fruits and olive oil. Similar components are included in the traditional Mediterranean diet defined a priori by Trichopoulou et $a l^{(3)}$. The beneficial effects of the Mediterranean diet have been widely examined ${ }^{(3,4,33-35)}$. Prospective studies such as the EPIC (European Prospective Investigation into Cancer and Nutrition)-Greek cohort have shown a decrease in mortality for all causes, CVD and cancer among subjects who follow this dietary pattern ${ }^{(3)}$. The ATTICA study found lower levels of C-reactive protein, IL-6, homocysteine and fibrinogen among those subjects with high adherence to the Mediterranean $\operatorname{diet}^{(33)}$. Recently, similar results have been obtained in the PREDIMED (Prevención con Dieta Mediterránea) study, a clinical trial of primary prevention of $\mathrm{CVD}^{(34)}$.

The current interest of nutritional epidemiology is the study of these and other dietary patterns and their relationship with several diseases or health conditions. But what is the role of different beverage consumption patterns on disease risk? And what is the contribution of beverage patterns to the health effects reported for several dietary patterns? Although these questions have not been disentangled yet, the role of different beverages on health outcomes has been analysed in several studies.

An increased risk of weight gain has been observed among subjects with high consumption of sugar-sweetened drinks ${ }^{(8,36)}$. Artificially sweetened soft drinks have been associated with increased risk for different types of cancer and mortality ${ }^{(37,38)}$. The consumption of cola, but not of other soft drinks, has also been related to lower bone mineral density in women ${ }^{(39)}$. Drinking patterns are associated with the risk of developing dental caries as well. Specifically, this risk is increased when soft drinks are predominant and is decreased when water, juices and milk are prevailing ${ }^{(40)}$. Moreover, an inverse relationship between juice consumption and metabolic syndrome prevalence has been reported ${ }^{(41)}$. Nevertheless, juice consumption has been related to increased weight gain among children with established overweight or at risk of developing it ${ }^{(42)}$. The consumption of low-fat dairy products has been inversely associated with hypertension ${ }^{(43)}$. Although coffee intake elevates blood pressure acutely, no association has been found between its intake and incident hypertension ${ }^{(44)}$. What is more, coffee consumption may reduce the risk of type 2 diabetes ${ }^{(45,46)}$. With regard to mortality, coffee has shown an inverse relationship with mortality due to cardiovascular and inflammatory diseases ${ }^{(47)}$.

However, the association between the consumption of a specific beverage and the consumption of others has not yet been clearly elucidated. Similarly and as far as we know, scarce data exist regarding the relationship between the consumption of a type of beverage and the adherence to a particular dietary pattern, and information is lacking on the characteristics of populations who consume different types of beverages ${ }^{(13,48-50)}$. Duffey and Popkin found that people with adherence to an unhealthy dietary pattern had also an unhealthy beverage pattern $^{(13)}$. However, whereas an inverse relationship between coffee consumption and adherence to the WDP and no significant association between its consumption and adherence to the MDP were found in the present SUN data analysis, coffee consumption has been inversely associated with the so-called Healthy Eating Index $(\mathrm{HEI})^{(48)}$. Forshee et al. also reported a positive association with the HEI for fruit drinks, carbonated soft drinks, tea and low-energy fruit drinks consumption, although the associations for tea and low-energy fruit drinks were statistically significant only among women ${ }^{(48)}$. In preschool children, a beverage pattern rich in fruit juice was associated with high HEI and high nutrient intake, whereas HEI was lower in pre-school children consuming more high-fat milk $^{(49)}$. In addition, in school-aged children, a beverage pattern rich in high-fat milk was related to a high micronutrient intake while soda and sweetened drinks were related to a lower micronutrient intake ${ }^{(49)}$. As far as the HEI is concerned, this index was higher in school-aged children with a beverage pattern rich in highfat milk ${ }^{(49)}$. It has also been observed that high-energy and soft/juice drinks consumption is lower among people with a healthier dietary pattern ${ }^{(50)}$, a finding which is consistent with our data. Beside this, moderate wine consumption has been related to a healthier dietary pattern whereas beer and spirits consumption has been inversely associated with salad consumption ${ }^{(51)}$. In the present study we also found an inverse linear trend between beer and spirits consumption and adherence to the MDP, whereas the linear trend for red wine consumption was not statistically significant. Moderate red 
wine consumption (around $25 \mathrm{~g} / \mathrm{d}$ ) was found in all quintiles of adherence.

The present results suggest that consumers of sugarsweetened beverages like soft drinks have a different food pattern from those who consume non-energy and diet beverages. This particular population is the same population whose subjects follow a WDP rich in fast foods, processed meals, processed pastries, refined grains, red meat, meat products and other food factors that have been shown to be associated with long-term weight gain ${ }^{(27)}$ with similar effect to that of sugar-sweetened beverages ${ }^{(8,35)}$. Therefore, the risk of adverse health outcomes might be increased among this particular population. This fact should be taken into account as one of the best ways to decrease energy intake in the implementation of health policy programmes targeting these particular populations, because food and beverage consumption seem to be closely linked. Thus, it seems that dietary patterns are predictors of beverage consumption and both dietary and beverage consumption patterns could be used to predict disease risk.

A potential limitation of the present study is related to the use of self-reported information. Although the validity and reliability of our semi-quantitative FFQ have been demonstrated $^{(14)}$, the validation study was conducted prior to the development of our cohort and did not include any of its members. Therefore, non-differential misclassification may exist and would be likely to bias the estimates towards the null. However, if that were the case, we would expect the true associations to be higher than those found in the present analyses.

For future analyses regarding diet-disease associations in epidemiological studies not only dietary patterns and lifestyle-related variables should be considered. Beverage patterns should be considered as well, because of the probability that all of them operate together on disease risk.

\section{Acknowledgements}

All authors declare having no conflicts of interest. The Spanish Ministry of Health (Fondo de Investigaciones Sanitarias, Projects PI042241, PI040233 \& PI050976 and RD06/0045) and the Navarra Regional Government (PI41/ 2005) are gratefully acknowledged for supporting the present study. A.S.-V. and E.T. undertook the statistical analyses and data interpretation, which were supervised by M.A.M.-G. M.A.M.-G. was also responsible for funding obtainment. All authors contributed to the elaboration and critical review for important intellectual content of the manuscript and gave final approval of the article. We are indebted to the participants of the SUN study for their continued cooperation and participation. We thank other members of the SUN Study Group: M. Seguí-Gómez, C. de la Fuente, J. de Irala, A. Alonso, M. Delgado-Rodríguez, M. Serrano-Martínez and J.A. Martínez.

\section{References}

1. Hu FB, Rimm E, Smith-Warner SA, Feskanich D, Stampfer MJ, Ascherio A, Sampson L \& Willett WC (1999) Reproducibility and validity of dietary patterns assessed with a food-frequency questionnaire. Am J Clin Nutr 69, 243-249.

2. Willett WC, Sacks F, Trichopoulou A, Drescher G, Ferro-Luzzi A, Helsing E \& Trichopoulos D (1995) Mediterranean diet pyramid: a cultural model for healthy eating. Am J Clin Nutr 61, Suppl. 6, 1402S-1406S.

3. Trichopoulou A, Costacou T, Bamia C \& Trichopoulos D (2003) Adherence to a Mediterranean diet and survival in a Greek population. N Engl J Med 348, 2599-2608.

4. Panagiotakos DB, Pitsavos C, Arvaniti F \& Stefanadis C (2007) Adherence to the Mediterranean food pattern predicts the prevalence of hypertension, hypercholesterolemia, diabetes and obesity, among healthy adults; the accuracy of the MedDietScore. Prev Med 44, 335-340.

5. Popkin BM, Armstrong LE, Bray GM, Caballero B, Frei B \& Willett WC (2006) A new proposed guidance system for beverage consumption in the United States. Am J Clin Nutr 83, 529-542.

6. French SA, Lin BH \& Guthrie JF (2003) National trends in soft drink consumption among children and adolescents age 6 to 17 years: prevalence, amounts, and sources, 1977-1978 to 1994-1998. J Am Diet Assoc 103, 2326-2331.

7. Comité de Nutrición de la Asociación Española de Pediatría (2003) Consumo de zumos de frutas y de bebidas refrescantes por niños y adolescentes en España. Implicaciones para la salud de su uso y abuso (Consumptions of fruit juices and beverages by Spanish children and teenagers: health implications of their poor use and abuse). An Pedriatr (Barc) 58, 584-593.

8. Bes-Rastrollo M, Sánchez-Villegas A, Gómez-Gracia E, Martínez JA, Pajares RM \& Martínez-González MA (2006) Predictors of weight gain in a Mediterranean cohort: the Seguimiento Universidad de Navarra Study. Am J Clin Nutr $\mathbf{8 3}, 362-370$.

9. Malik VS, Schulze MB \& Hu FB (2006) Intake of sugarsweetened beverages and weight gain: a systematic review. Am J Clin Nutr 84, 274-288.

10. Vartanian LR, Schwartz MB \& Brownell KD (2007) Effects of soft drink consumption on nutrition and health: a systematic review and meta-analysis. Am J Public Health 97, 667-675.

11. Kay CD, Kris-Etherton PM \& West SG (2006) Effects of antioxidant-rich foods on vascular reactivity: review of the clinical evidence. Curr Atheroscler Rep 8, 510-522.

12. Hodge AM, English DR, O'Dea K \& Giles GG (2006) Alcohol intake, consumption pattern and beverage type, and the risk of type 2 diabetes. Diabet Med 23, 690-697.

13. Duffey KJ \& Popkin BM (2006) Adults with healthier dietary patterns have healthier beverage patterns. J Nutr 136, 2901-2907.

14. Martin-Moreno JM, Boyle P, Gorgojo L, Maisonneuve P, Fernandez-Rodriguez JC, Salvini S \& Willett WC (1993) Development and validation of a food frequency questionnaire in Spain. Int J Epidemiol 22, 512-519.

15. Mataix J (2003) Tabla de Composición de Alimentos (Food Composition Tables), 4th ed. Granada: Universidad de Granada.

16. Moreiras O (2003) Tablas de Composición de Alimentos (Food Composition Tables), 7th ed. Madrid: Ediciones Madrid Pirámide.

17. Pett MA, Lackey NR \& Sullivan JJ (2003) Making Sense of Factor Analysis: The Use of Factor Analysis for Instrument Development in Health Care Research. Thousand Oaks, CA: Sage. 
18. Hair JF, Anderson RE, Tatham RL \& Black WC (1995) Multivariate Data Analysis with Readings, 4th ed. Englewood Cliffs, NJ: Prentice Hall.

19. Chasan-Taber S, Rimm EB, Stampfer MJ, Spiegelman D, Colditz GA, Giovannucci E, Ascherio A \& Willett WC (1996) Reproducibility and validity of a self-administered physical activity questionnaire for male health professionals. Epidemiology 7, 81-86.

20. Martínez-González MA, López-Fontana C, Varo JJ, SánchezVillegas A \& Martínez JA (2005) Validation of the Spanish version of the physical activity questionnaire used in the Nurses' Health Study and Health Professionals' Follow-up Study. Public Health Nutr 8, 920-927.

21. Sánchez-Villegas A, Delgado-Rodríguez M, MartínezGonzález MA \& De Irala-Estévez J, Seguimiento Universidad de Navarra group (2003) Gender, age, socio-demographic and lifestyle factors associated with major dietary patterns in the Spanish Project SUN (Seguimiento Universidad de Navarra). Eur J Clin Nutr 57, 285-292.

22. Esmaillzadeh A, Kimiagar M, Mehrabi Y, Azadbakht L, Hu FB \& Willett WC (2007) Dietary patterns, insulin resistance, and prevalence of the metabolic syndrome in women. Am J Clin Nutr 85, 910-918.

23. Fung TT, Hu FB, Holmes MD, Rosner BA, Hunter DJ, Colditz GA \& Willett WC (2005) Dietary patterns and the risk of postmenopausal breast cancer. Int J Cancer 116, 116-121.

24. Fung $\mathrm{T}$, Hu FB, Fuchs C, Giovannucci E, Hunter DJ, Stampfer MJ, Colditz GA \& Willett WC (2003) Major dietary patterns and the risk of colorectal cancer in women. Arch Intern Med 163, 309-314.

25. Fung TT, Stampfer MJ, Manson JE, Rexrode KM, Willett WC $\&$ Hu FB (2004) Prospective study of major dietary patterns and stroke risk in women. Stroke 35, 2014-2019.

26. Hu FB, Rimm EB, Stampfer MJ, Ascherio A, Spiegelman D \& Willett WC (2000) Prospective study of major dietary patterns and risk of coronary heart disease in men. $A m J$ Clin Nutr 72, 912-921.

27. Fung TT, Willett WC, Stampfer MJ, Manson JE \& Hu FB (2001) Dietary patterns and the risk of coronary heart disease in women. Arch Intern Med 161, 1857-1862.

28. Schulze MB, Fung TT, Manson JE, Willett WC \& Hu FB (2006) Dietary patterns and changes in body weight in women. Obesity 14, 1444-1453.

29. van Dam RM, Rimm EB, Willett WC, Stampfer MJ \& Hu FB (2002) Dietary patterns and risk for type 2 diabetes mellitus in US men. Ann Intern Med 136, 201-209.

30. Fung TT, Schulze M, Manson JE, Willett WC \& Hu FB (2004) Dietary patterns, meat intake, and the risk of type 2 diabetes in women. Arch Intern Med 164, 2235-2240.

31. Lopez-Garcia E, Schulze MB, Fung TT, Meigs JB, Rifai N, Manson JE \& Hu FB (2004) Major dietary patterns are related to plasma concentrations of markers of inflammation and endothelial dysfunction. Am J Clin Nutr 80, 1029-1035.

32. Fung TT, Rimm EB, Spiegelman D, Rifai N, Tofler GH, Willett WC \& Hu FB (2001) Association between dietary patterns and plasma biomarkers of obesity and cardiovascular disease risk. Am J Clin Nutr 73, 61-67.

33. Chrysohoou C, Panagiotakos DB, Pitsavos C, Das UN \& Stefanadis C (2004) Adherence to the Mediterranean diet attenuates inflammation and coagulation process in healthy adults: the ATTICA study. J Am Coll Cardiol 44, 152-158.

34. Estruch R, Martinez-Gonzalez MA, Corella D et al.; PREDIMED Study Investigators (2004) Effects of a
Mediterranean-style diet on cardiovascular risk factors: a randomized trial. Ann Intern Med 145, 1-11.

35. Martínez-González MA \& Sánchez-Villegas A (2004) The emerging role of Mediterranean diets in cardiovascular epidemiology: monounsaturated fats, olive oil, red wine or the whole pattern? Eur J Epidemiol 19, 9-13.

36. Malik VS, Schulze MB \& Hu FB (2006) Intake of sugarsweetened beverages and weight gain: a systematic review. Am J Clin Nutr 84, 274-288.

37. Larsson SC, Bergkvist L \& Wolk A (2006) Consumption of sugar and sugar-sweetened foods and the risk of pancreatic cancer in a prospective study. Am J Clin Nutr 84, $1171-1176$.

38. Paganini-Hill A, Kawas CH \& Corrada MM (2007) Nonalcoholic beverage and caffeine consumption and mortality: The Leisure World Cohort Study. Prev Med 44, 305-310.

39. Tucker KL, Morita K, Qiao N, Hannan MT, Cupples LA \& Kiel DP (2006) Colas, but not other carbonated beverages, are associated with low bone mineral density in older women: The Framingham Osteoporosis Study. Am J Clin Nutr 84, 936-942.

40. Sohn W, Burt BA \& Sowers MR (2006) Carbonated soft drinks and dental caries in the primary dentition. J Dent Res $\mathbf{8 5}, 262-266$.

41. Yoo S, Nicklas T, Baranowski T, Zakeri IF, Yang SJ, Srinivasan SR \& Berenson GS (2004) Comparison of dietary intakes associated with metabolic syndrome risk factors in young adults: the Bogalusa Heart Study. Am J Clin Nutr 80, 841-848.

42. Faith MS, Dennison BA, Edmunds LS \& Stratton HH (2006) Fruit juice intake predicts increased adiposity gain in children from low-income families: weight statusby-environment interaction. Pediatrics 118, 2066-2075.

43. Alonso A, Beunza JJ, Delgado-Rodriguez M, Martinez JA \& Martinez-Gonzalez MA (2005) Low-fat dairy consumption and reduced risk of hypertension: the Seguimiento Universidad de Navarra (SUN) cohort. Am J Clin Nutr 82, 972-979.

44. Winkelmayer WC, Stampfer MJ, Willett WC \& Curhan GC (2005) Habitual caffeine intake and the risk of hypertension in women. JAMA 294, 2330-2335.

45. van Dam RM \& Hu FB (2005) Coffee consumption and risk of type 2 diabetes: a systematic review. JAMA 294, 97-104.

46. Iso H, Date C, Wakai K, Fukui M \& Tamakoshi A, JACC Study Group (2006) The relationship between green tea and total caffeine intake and risk for self-reported type 2 diabetes among Japanese adults. Ann Intern Med 144, 554-562.

47. Andersen LF, Jacobs DR Jr, Carlsen MH \& Blomhoff $\mathrm{R}$ (2006) Consumption of coffee is associated with reduced risk of death attributed to inflammatory and cardiovascular diseases in the Iowa Women's Health Study. Am J Clin Nutr 83, 1039-1046.

48. Forshee RA \& Storey ML (2006) Demographics, not beverage consumption, is associated with diet quality. Int J Food Sci Nutr 57, 494-511.

49. Larowe TL, Moeller SM \& Adams AK (2007) Beverage patterns, diet quality, and body mass index of US preschool and school-aged children. J Am Diet Assoc 107, 1124-1133.

50. Popkin BM, Barclay DV \& Nielsen SJ (2005) Water and food consumption patterns of US adults from 1999 to 2001. Obes Res 13, 2146-2152.

51. Tjonneland A, Gronbaek M, Stripp C \& Overvad K (1999) Wine intake and diet in a random sample of 48763 Danish men and women. Am J Clin Nutr 69, 49-54. 